\title{
Adaptive Path Following Primal Dual Interior Point Methods for Shape Optimization of Linear and Nonlinear Stokes Flow Problems
}

\author{
Ronald H.W. Hoppe ${ }^{1,2}$, Christopher Linsenmann ${ }^{1,2}$, and Harbir Antil ${ }^{1}$ \\ 1 University of Houston, Department of Mathematics \\ (http://www. math.uh.edu/ rohop/) \\ 2 University of Augsburg, Institute for Mathematics \\ (http://scicomp.math.uni-augsburg.de)
}

Summary. We are concerned with structural optimization problems in CFD where the state variables are supposed to satisfy a linear or nonlinear Stokes system and the design variables are subject to bilateral pointwise constraints. Within a primaldual setting, we suggest an all-at-once approach based on interior-point methods. The discretization is taken care of by Taylor-Hood elements with respect to a simplicial triangulation of the computational domain. The efficient numerical solution of the discretized problem relies on adaptive path-following techniques featuring a predictor-corrector scheme with inexact Newton solves of the KKT system by means of an iterative null-space approach. The performance of the suggested method is documented by several illustrative numerical examples.

\section{Introduction}

Simplified problems in shape optimization have already been addressed by Bernoulli, Euler, Lagrange and Saint-Venant. However, it became its own discipline during the second half of the last century when the rapidly growing performance of computing platforms and the simultaneously achieved significant improvement of algorithmic tools enabled the appropriate treatment of complex problems (cf. $[1 ; 3 ; 6 ; 9 ; 13 ; 14 ; 15]$ and the references therein). The design criteria in shape optimization are determined by a goal oriented operational behavior of the devices and systems under consideration and typically occur as nonlinear, often non convex, objective functionals which depend on the state variables describing the operational mode and the design variables determining the shape. The state variables often satisfy partial differential equations or systems thereof representing the underlying physical laws. Technological aspects are taken into account by constraints on the state and/or design variables which may occur both as equality and inequality constraints in the model. 
Shape optimization problems associated with fluid flow problems play an important role in a wide variety of engineering applications [13]. A typical setting is the design of the geometry of the container of the fluid, e.g., a channel, a reservoir, or a network of channels and reservoirs such that a desired flow velocity and/or pressure profile is achieved. The solution of the problem amounts to the minimization of an objective functional that depends on the state variables (velocity, pressure) and on the design variables which determine the geometry of the fluid filled domain. The state variables are supposed to satisfy the underlying fluid mechanical equations, and there are typically constraints on the design variables which restrict the shape of the fluid filled domain to that what is technologically feasible.

The typical approach to shape optimization problems relies on a separate treatment of the design issue and the underlying state equation what is called alternate approximation in [1]: For a given initial design the state equation is solved, followed by a sensitivity analysis that leads to an update of the design variables. This process is iteratively repeated until convergence. Moreover, many methods, e.g., those based on the concept of shape derivatives $[6 ; 15]$, only use first order information by employing gradient type techniques. In this paper, we focus on a so-called all-at-once approach where the numerical solution of the discretized state equation is an integral part of the optimization routine (cf, e.g., $[4 ; 5 ; 10 ; 12])$. Moreover, we use second order information by means of primal-dual interior-point methods. In particular, we consider an adaptive path-following technique for the shape optimization of stationary flow problems as described by a linear or nonlinear Stokes system in channels where the objective is to design the lateral walls such that a desired velocity and/or pressure profile is obtained. The design variables are chosen as the control points of a Bézier curve representation of the lateral walls.

The paper is organized as follows: Section 2 is devoted to the setup of the shape optimization problem including its finite element discretization by Taylor-Hood elements. In section 3, we focus on the primal-dual interiorpoint approach and a path-following predictor-corrector type continuation method with an adaptive choice of the continuation parameter. Finally, in section 4 we illustrate the application of the algorithm for the design of a channel with a backward facing step assuming a linear Stokes regime and for the shape optimization of the inlet and outlet boundaries of the ducts of an electrorheological shock absorber, where the states satisfy a nonlinear Stokes equation.

\section{Shape optimization of stationary Stokes flow}

We consider Stokes flow in a bounded domain $\Omega(\alpha) \subset \mathbb{R}^{2}$ with boundary $\Gamma(\alpha)=\Gamma_{\text {in }}(\alpha)+\Gamma_{\text {out }}(\alpha)+\Gamma_{\text {lat }}(\alpha)$ consisting of the inflow, the outflow and the lateral boundaries with $\mathbf{n}$ and $\mathbf{t}$ denoting the outward unit normal and unit 
tangential vector, respectively. Here, $\alpha=\left(\alpha_{1}, \cdots, \alpha_{m}\right)^{T} \in \mathbb{R}^{m}$ is the vector of design variables which are chosen as the Bézier control points of a Bézier curve representation of $\Gamma(\alpha)$ and which are subject to upper and lower bounds $\alpha_{i}^{\min }, \alpha_{i}^{\max }, 1 \leq i \leq m$. The state variables are the velocity $\mathbf{u}$ and the pressure $p$. Given desired velocity and pressure profiles $\mathbf{u}^{\mathbf{d}}$ and $p^{d}$, an inflow $u_{\text {in }}$ at the inflow boundary $\Gamma_{i n}(\alpha)$ and weighting factors $\kappa_{i} \geq 0,1 \leq i \leq 2, \kappa_{1}+\kappa_{2}>0$, the shape optimization problem can be stated as follows:

$$
\begin{array}{ll}
\text { minimize } & J(\mathbf{u}, p, \alpha)=\frac{\kappa_{1}}{2} \int_{\Omega(\alpha)}\left|\mathbf{u}-\mathbf{u}^{\mathbf{d}}\right|^{2} d x+\frac{\kappa_{2}}{2} \int_{\Omega(\alpha)}\left|p-p^{d}\right|^{2} d x, \\
\text { subject to } \quad-\nabla \cdot \boldsymbol{\sigma}(\mathbf{u})=0 \quad \text { in } \Omega(\alpha) \\
\nabla \cdot \mathbf{u}=0 \quad \text { in } \Omega(\alpha) \\
\boldsymbol{\sigma}(\mathbf{u})=-p \mathbf{I}+g(\mathbf{u}, \mathbf{D}(\mathbf{u})) \mathbf{D}(\mathbf{u}) \\
\mathbf{n} \cdot \mathbf{u}=u_{\text {in }} \quad \text { on } \Gamma_{\text {in }}(\alpha) \\
\mathbf{n} \cdot \mathbf{u}=0 \quad \text { on } \Gamma_{\text {lat }}(\alpha) \\
\mathbf{t} \cdot \mathbf{u}=0 \quad \text { on } \Gamma(\alpha), \\
\alpha_{i}^{\text {min }} \leq \alpha_{i} \leq \alpha_{i}^{\text {max }}, 1 \leq i \leq m .
\end{array}
$$

We note that in the constitutive equation (1c) the tensor $\mathbf{D}(\mathbf{u})$ stands for the rate of deformation tensor $\mathbf{D}(\mathbf{u}):=\left(\nabla \mathbf{u}+(\nabla \mathbf{u})^{T}\right) / 2$ and $g(\mathbf{u}, \mathbf{D}(\mathbf{u}))$ denotes the viscosity function which is given by $g(\mathbf{u}, \mathbf{D}(\mathbf{u}))=\nu$ for linear Stokes flow and depends nonlinearly on $\mathbf{u}, \mathbf{D}(\mathbf{u})$ in the nonlinear regime.

We choose $\hat{\alpha} \in K$ as a reference design and refer to $\hat{\Omega}:=\Omega(\hat{\alpha})$ as the associated reference domain. Then, the actual domain $\Omega(\alpha)$ can be obtained from the reference domain $\hat{\Omega}$ by means of an isomorphism

$$
\begin{aligned}
\Omega(\alpha) & =\Phi(\hat{\Omega} ; \alpha), \\
\Phi(\hat{x} ; \alpha) & =\left(\Phi_{1}(\hat{x} ; \alpha), \Phi_{2}(\hat{x} ; \alpha)\right)^{T}, \hat{x}=\left(\hat{x}_{1}, \hat{x}_{2}\right)^{T}
\end{aligned}
$$

with continuous components $\Phi_{i}, 1 \leq i \leq 2$. Due to the reference domain, finite element approximations of (1) can be performed with respect to $\hat{\Omega}$ without being forced to remesh any time the design parameters are changed.

We introduce $\left(\mathcal{T}_{h}(\hat{\Omega})\right)_{\mathbb{N}}$ as a shape regular family of simplicial triangulations of $\hat{\Omega}$. In view of (2), these triangulations induce an associated family $\left(\mathcal{T}_{h}(\Omega(\alpha))\right)_{\mathbb{N}}$ of simplicial triangulations of the actual physical domains $\Omega(\alpha)$. For the discretization of the velocity $\mathbf{u}$ and the pressure $p$ we use Taylor-Hood $\mathrm{P} 2 / \mathrm{P} 1$ elements. We refer to $\mathbf{u}_{h}^{d} \in \mathbb{R}^{n_{1}}$ and $p_{h}^{d} \in \mathbb{R}^{n_{2}}$ as the vectors representing the $L^{2}$-projections of $\mathbf{u}^{\mathrm{d}}, p$ onto the respective finite element spaces giving rise to the discrete objective functional

$$
J_{h}\left(\mathbf{y}_{h}, \alpha\right):=\frac{\kappa_{1}}{2}\left(\mathbf{u}_{h}-\mathbf{u}_{h}^{d}\right)^{T} I_{1, h}(\alpha)\left(\mathbf{u}_{h}-\mathbf{u}_{h}^{d}\right)+\frac{\kappa_{2}}{2} p_{h}^{T} I_{2, h}(\alpha) p_{h},
$$

where $\mathbf{y}_{h}:=\left(\mathbf{u}_{h}, p_{h}\right)^{T}$ and $I_{i, h}(\alpha), 1 \leq i \leq 2$, are the associated mass matrices. Further, denoting by 


$$
S_{h}\left(\mathbf{y}_{h}, \alpha\right):=\left(\begin{array}{cc}
A_{h}\left(\mathbf{u}_{h}, \alpha\right) & B_{h}^{T}(\alpha) \\
B_{h}(\alpha) & 0
\end{array}\right)\left(\begin{array}{l}
\mathbf{u}_{h} \\
p_{h}
\end{array}\right)=\left(\begin{array}{l}
\mathbf{g}_{1, h} \\
\mathbf{g}_{2, h}
\end{array}\right)=: \mathbf{g}_{h},
$$

the Taylor-Hood approximation of the Stokes system (1a), the discretized shape optimization problem can be stated as

$$
\begin{array}{ll}
\operatorname{minimize} & J_{h}\left(\mathbf{y}_{h}, \alpha\right), \\
\text { subject to } & S_{h}\left(\mathbf{y}_{h}, \alpha\right)=\mathbf{g}_{h}, \\
& \alpha_{i}^{\text {min }} \leq \alpha_{i} \leq \alpha_{i}^{\text {max }}, 1 \leq i \leq m .
\end{array}
$$

For notational convenience, in the sequel we will drop the discretization subindex $h$.

\section{Path-following primal-dual interior-point method}

We use a primal-dual interior-point method where the inequality constraints (4c) are coupled by logarithmic barrier functions with a barrier parameter $\beta=1 / \mu>0, \mu \rightarrow \infty$, resulting in the following parameterized family of minimization subproblems

$$
\inf _{\mathbf{y}, \alpha} B(\mathbf{y}, \alpha, \mu):=J(\mathbf{y}, \alpha)-\frac{1}{\mu} \sum_{i=1}^{m}\left[\ln \left(\alpha_{i}-\alpha_{i}^{\min }\right)+\ln \left(\alpha_{i}^{\max }-\alpha_{i}\right)\right]
$$

subject to (4b). Coupling (4b) by a Lagrange multiplier $\boldsymbol{\lambda}=\left(\boldsymbol{\lambda}_{\mathbf{u}}, \lambda_{p}\right)^{T}$, we are led to the saddle point problem

$$
\inf _{\mathbf{y}, \alpha} \sup _{\boldsymbol{\lambda}} L^{(\mu)}(\mathbf{y}, \boldsymbol{\lambda}, \alpha)=B^{(\mu)}(\mathbf{y}, \alpha)+\langle S(\mathbf{y}, \alpha)-\mathbf{g}, \boldsymbol{\lambda}\rangle .
$$

The central path $\mu \longmapsto x(\mu):=(\mathbf{y}(\mu), \boldsymbol{\lambda}(\mu), \alpha(\mu))^{T}$ is given as the solution of the nonlinear system

$$
F(x(\mu), \mu)=\left(\begin{array}{c}
L_{\mathbf{y}}^{(\mu)}(\mathbf{y}, \boldsymbol{\lambda}, \alpha) \\
L_{\lambda}^{(\mu)}(\mathbf{y}, \boldsymbol{\lambda}, \alpha) \\
L_{\alpha}^{(\mu)}(\mathbf{y}, \boldsymbol{\lambda}, \alpha)
\end{array}\right)=0
$$

which represents the first order necessary optimality conditions for (5).

For the solution of (7) we use an adaptive path-following predictor-corrector strategy following strategies developed in [7].

Predictor Step: The predictor step relies on tangent continuation along the trajectory of the Davidenko equation

$$
F_{\mathbf{x}}(\mathbf{x}(\mu), \mu) \mathbf{x}^{\prime}(\mu)=-F_{\mu}(\mathbf{x}(\mu), \mu) .
$$


Given some approximation $\tilde{\mathbf{x}}\left(\mu_{k}\right)$ at $\mu_{k}>0$, compute $\tilde{\mathbf{x}}^{(0)}\left(\mu_{k+1}\right)$, where $\mu_{k+1}=\mu_{k}+\Delta \mu_{k}^{(0)}$, according to

$$
\begin{aligned}
F_{x}\left(\tilde{\mathbf{x}}\left(\mu_{k}\right), \mu_{k}\right) \delta \mathbf{x}\left(\mu_{k}\right) & =-F_{\mu}\left(\tilde{\mathbf{x}}\left(\mu_{k}\right), \mu_{k}\right), \\
\tilde{\mathbf{x}}^{(0)}\left(\mu_{k+1}\right) & =\tilde{\mathbf{x}}\left(\mu_{k}\right)+\Delta \mu_{k}^{(0)} \delta \mathbf{x}\left(\mu_{k}\right) .
\end{aligned}
$$

We use $\Delta \mu_{0}^{(0)}=\Delta \mu_{0}$ for some given initial step size $\Delta \mu_{0}$, whereas for $k \geq 1$ the predicted step size $\Delta \mu_{k}^{(0)}$ is chosen by

$$
\Delta \mu_{k}^{(0)}:=\left(\frac{\left\|\Delta \mathbf{x}^{(0)}\left(\mu_{k}\right)\right\|}{\left\|\tilde{\mathbf{x}}\left(\mu_{k}\right)-\tilde{\mathbf{x}}^{(0)}\left(\mu_{k}\right)\right\|} \frac{\sqrt{2}-1}{2 \Theta\left(\mu_{k}\right)}\right)^{1 / 2} \Delta \mu_{k-1},
$$

where $\Delta \mu_{k-1}$ is the computed continuation step size, $\Delta \mathbf{x}^{(0)}\left(\mu_{k}\right)$ is the first Newton correction (see below), and $\Theta\left(\mu_{k}\right)<1$ is the contraction factor associated with a successful previous continuation step.

Corrector step: As a corrector, we use Newton's method applied to

$$
F\left(\mathbf{x}\left(\mu_{k+1}\right), \mu_{k+1}\right)=0
$$

with $\tilde{\mathbf{x}}^{(0)}\left(\mu_{k+1}\right)$ from (9) as a start vector. In particular, for $\ell \geq 0$ and $j_{\ell} \geq 0$ we compute $\Delta x^{\left(j_{\ell}\right)}\left(\mu_{k+1}\right)$ according to

$$
F^{\prime}\left(\tilde{x}^{\left(j_{\ell}\right)}\left(\mu_{k+1}\right), \mu_{k+1}\right) \Delta x^{\left(j_{\ell}\right)}\left(\mu_{k+1}\right)=-F\left(\tilde{x}^{\left(j_{\ell}\right)}\left(\mu_{k+1}\right), \mu_{k+1}\right)
$$

and $\overline{\Delta x}^{(j \ell)}\left(\mu_{k+1}\right)$ as the associated simplified Newton correction

$F^{\prime}\left(\tilde{x}^{\left(j_{\ell}\right)}\left(\mu_{k+1}\right), \mu_{k+1}\right) \overline{\Delta x}^{\left(j_{\ell}\right)}\left(\mu_{k+1}\right)=-F\left(\tilde{x}^{\left(j_{\ell}\right)}\left(\mu_{k+1}\right)+\Delta x^{\left(j_{\ell}\right)}\left(\mu_{k+1}\right), \mu_{k+1}\right)$.

We monitor convergence of Newton's method by means of

$$
\Theta^{\left(j_{\ell}\right)}\left(\mu_{k+1}\right):=\left\|\overline{\Delta x}^{\left(j_{\ell}\right)}\left(\mu_{k+1}\right)\right\| /\left\|\Delta x^{\left(j_{\ell}\right)}\left(\mu_{k+1}\right)\right\| .
$$

In case of successful convergence, we accept the current step size and proceed with the next continuation step. However, if the monotonicity test

$$
\Theta^{\left(j_{\ell}\right)}\left(\mu_{k+1}\right)<1
$$

fails for some $j_{\ell} \geq 0$, the continuation step has to be repeated with the reduced step size

$$
\Delta \mu_{k}^{(\ell+1)}:=\left(\frac{\sqrt{2}-1}{g\left(\Theta^{\left(j_{\ell}\right)}\right)}\right)^{1 / 2} \Delta \mu_{k}^{(\ell)} \quad, \quad g(\Theta):=\sqrt{\Theta+1}-1
$$

until we either achieve convergence or for some prespecified lower bound $\Delta \mu_{\min }$ observe

$$
\Delta \mu_{k}^{(\ell+1)}<\Delta \mu_{\min } .
$$

In the latter case, we stop the algorithm and report convergence failure.

The Newton steps are realized by an inexact Newton method featuring righttransforming iterations (cf., e.g., $[10 ; 12])$. The derivatives occurring in the KKT conditions and the Hessians are computed by automatic differentiation [8]. 


\section{Numerical simulation results}

As a first example, we consider linear Stokes flow with viscosity $\nu=1$ and given inflow $u_{i n}$ in a channel with a backward facing step (cf. Fig. 1 (top)). The initial shape corresponds to the straight line, whereas the desired velocity profile $\mathbf{u}^{\mathbf{d}}$ has been chosen according to a shape represented by the dotted line. We have used a total of six Bézier control points with given lower and upper bounds. The results are displayed in Fig. 1. For details concerning the convergence history we refer to [2].

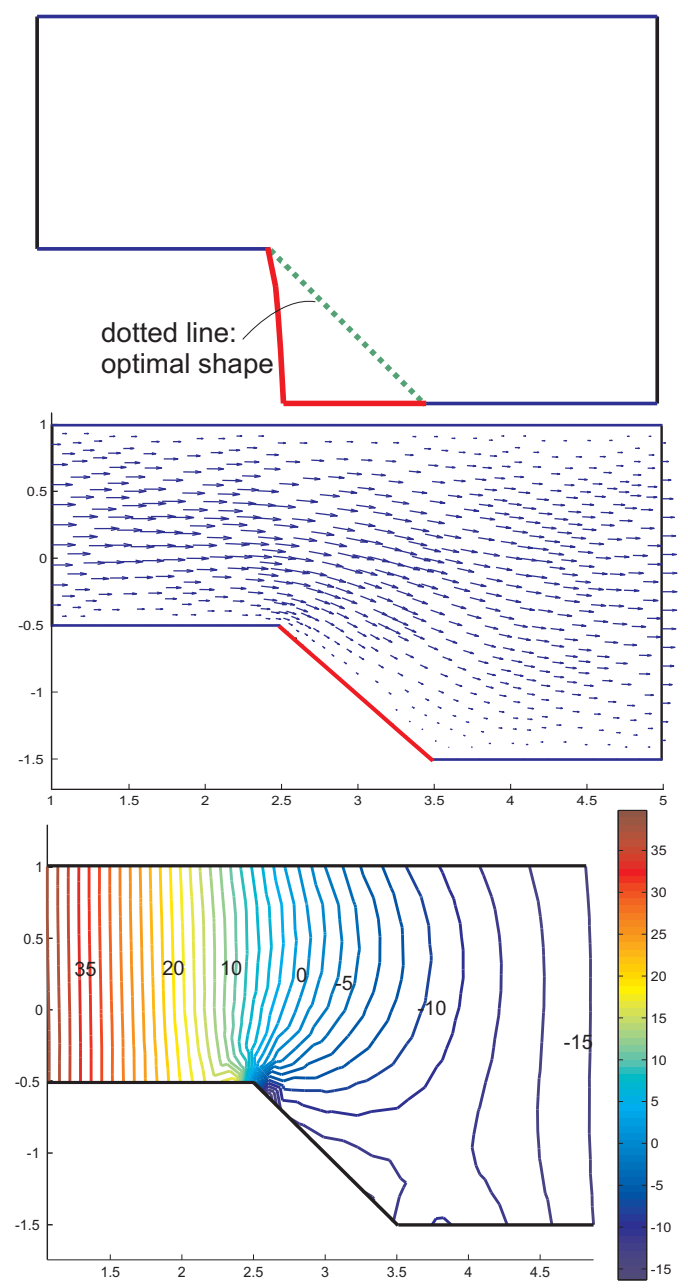

Fig. 1. Backward facing step with final shape (top) and computed velocity field (middle) and pressure distribution (bottom). 
As an example for a shape optimization problem associated with nonlinear Stokes flow, we consider the optimization of the inlet and outlet boundaries of the ducts in an electrorheological shock absorber (cf. Fig. 2 (left). Such shock absorbers are based on an electrorheological fluids (ERF). An ERF is a suspension of small electrically polarizable particles dissolved in nonconducting liquids which under the influence of an outer electric field changes its viscosity within a few milliseconds in a reversible way. The viscosity function in (1c) is of the form $g(I(\mathbf{u}),|\mathbf{E}|)$, where $I(\mathbf{u})$ is the second invariant of the rate of strain tensor and $|\mathbf{E}|$ stands for the electric field strength (for details see [11]). The issue is to avoid too large pressure fluctuations at the boundaries of the duct both in the compression and the rebound mode. We have chosen a desired pressure profile $p^{d}$ and used Bézier curve representations of the inlet and outlet boundaries as illustrated in Fig. 2 (middle). A computed optimal shape of the outlet boundary in the rebound mode is shown in Fig. 2 (right). For details we refer to [10].
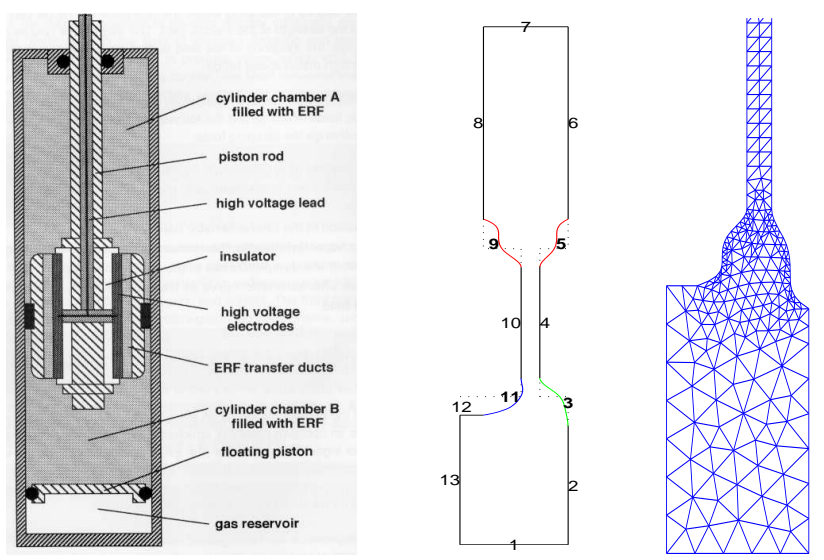

Fig. 2. Electrorheological shock absorber (left), Bézier curve representation of the inlet and outlet boundary of the right part of the fluid chamber (middle), and optimal design of the outlet boundary in rebound mode (right)

Acknowledgements. The first two authors acknowledge support by the NSF under Grant-No. DMS-0511611 and by the German National Science Foundation within the Priority program SPP1253 'Optimization with Partial Differential Equations'. The work of the third author is supported by the Texas Computational and Learning Center TLC2. 


\section{References}

[1] Allaire, G. (2002). Shape Optimization by the Homogenization Method. Springer, Berlin-Heidelberg-New York.

[2] Antil, H., Hoppe, R.H.W., and Linsenmann, C. (2007). Path-following primal-dual interior-point methods for shape optimization of stationary flow problems. to appear in Journal of Numerical Mathematics.

[3] Bendsøe, M.P. (1995). Optimization of Structural Topology, Shape, and Material. Springer, Berlin-Heidelberg-New York.

[4] Biros, G., and Ghattas, O. (2004). Parallel Lagrange-Newton-KrylovSchur methods for PDE-constrained optimization. part $i$ : the KrylovSchur solver. to appear in SIAM J. Sci. Comp.

[5] Biros, G., and Ghattas, O. (2004). Parallel Lagrange-Newton-KrylovSchur methods for PDE-constrained optimization. part ii: the LagrangeNewton solver and its application to optimal control of staedy viscous flows. to appear in SIAM J. Sci. Comp.

[6] Delfour, M.C., and Zolesio, J.P. (2001). Shapes and Geometries: Analysis, Differential Calculus and Optimization. SIAM, Philadelphia.

[7] Deuflhard, P. (2004). Newton Methods for Nonlinear Problems. Affine Invariance and Adaptive Algorithms. Springer, Berlin-Heidelberg-New York.

[8] Griewank, A. (2000). Evaluating Derivatives, Principles and Techniques of Automatic Differentiation. SIAM, Phildelphia.

[9] Haslinger, J., and Mäkinen, R.A.E. (2004). Introduction to Shape Optimization: Theory, Approximation, and Computation. SIAM, Philadelphia.

[10] Hoppe, R.H.W., Linsenmann, C., and Petrova, S.I. (2006). Primal-dual Newton methods in structural optimization. Comp. Visual. Sci. 9, 71-87.

[11] Hoppe, R.H.W., and Litvinov, W.G. (2004). Problems on electrorheological fluid flows. Communications in Pure and Applied Analysis, 3, 809-848.

[12] Hoppe, R.H.W., and Petrova, S.I. (2004). Primal-dual Newton interior point methods in shape and topology optimization. Numerical Linear Algebra with Applications 11, 413-429.

[13] Mohammadi, B. and Pironneau, O. (2001). Applied Shape Optimization for Fluids. Oxford University Press, Oxford.

[14] Rozvany, G. (1989). Structural Design via Optimality Criteria. Kluwer, Dordrecht.

[15] Sokolowski, J., and Zolesio, J.P. (1992). Introduction to Shape Optimization. Springer, Berlin-Heidelberg-New York, 1992. 
